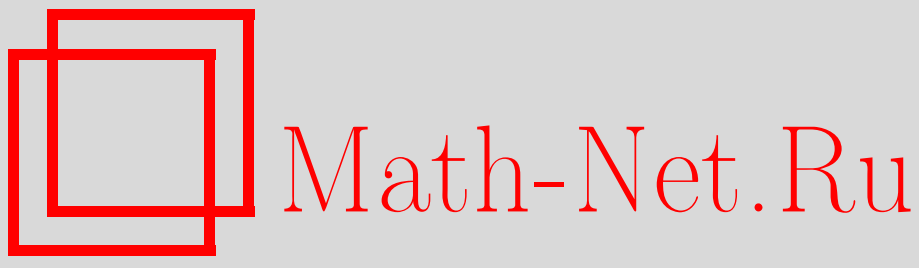

Л. А. Камынин, Б. Н. Химченко, О слабом (алгебраическом) принципе экстремума для параболической системы 2-го порядка, Изв. РАН. Сер. матем., 1997, том 61, выпуск 5, 35-62

DOI: https://doi.org/10.4213/im150

Использование Общероссийского математического портала Math-Net.Ru подразумевает, что вы прочитали и согласны с пользовательским соглашением

http://www.mathnet.ru/rus/agreement

Параметры загрузки:

IP : 54.162 .27 .143

26 апреля 2023 г., 15:04:54 
УДК 517.96

\author{
Л. И. Камынин, Б.Н. Химченко
}

\title{
О слабом (алгебраическом) принципе экстремума для параболической системы 2-го порядка
}

\begin{abstract}
Вводится понятие слабого “алгебраического” принципа экстремума (САПЭ) для параболических систем 2-го порядка, основанного на представлении матриц (коэффициентов системы) в виде суммы матриц, подобных диагональным, и нильпотентных матриц и сведении системы к одному уравнению. Справедливость САПЭ доказана для достаточно широкого класса параболических систем 2-го порядка с "полным перемешиванием". САПЭ применен к доказательству единственности решения I-ой краевой задачи для рассматриваемых параболических систем.

Библиография: 10 наименований.
\end{abstract}

Известно, что для параболического уравнения 2-го порядка с неотрицательной характеристической формой имеет место (см. [1]-[6]) в той или иной форме слабый принщип экстремума, играющий важную роль в качественной теории таких уравнений. Вопрос о справедливости слабого принщипа экстремума для параболических систем также поднимался в ряде работ (см., например, [7]-[9]), причем чаше исследовались системы с перемешиванием лишь в младших членах, не содержаших производных. В нашей статье вопрос о слабом принщипе экстремума рассматривается для параболических систем 2-го порядка с полным перемешиванием членов при новой трактовке самой постановки принципа экстремума. Вводится понятие “алгебраического" слабого принщипа экстремума, основанного на разложении матриц (коэффициентов системы) как в матрицы простой структуры (подобные диагональным), так и в нильпотентные матрицы (см. лемму 1) и сведении матрично-векторных уравнений к системе скалярных уравнений специального вида (см. лемму 2). Основной идеей при этом является сведение принципа экстремума для параболической системы 2-го порядка к обычному принципу экстремума для одного параболического уравнения 2-го порядка.

В статье доказана теорема 1 о слабом принципе экстремума в его "алгебраической” постановке для достаточно широкого класса параболических систем 2-го порядка с "полным перемешиванием". В теоремах 2-4 приводятся приложения установленного слабого принципа экстремума к проблеме о единственности решения I-ой краевой задачи для параболических систем 2-го порядка.

Пусть

$$
G \subset\{0<t<T<+\infty\} \subset \mathbb{R}_{x, t}^{n+1}, \quad n \geqslant 1,
$$

- ограниченная область, для которой

$$
\partial G=\gamma_{0}(G) \cup \Sigma(G) \cup \gamma(G),
$$


где нижняя крышка

$$
\gamma_{0}(G)=\operatorname{int}(\partial G \cap\{t=0\}) \subset \mathbb{R}_{x}^{n}
$$

является областью на гиперплоскости $\{t=0\}$, верхняя крышка

$$
\gamma(G)=\operatorname{int}(\bar{G} \cap\{t=T>0\}) \subset \mathbb{R}_{x}^{n}
$$

является областью на гиперплоскости $\{t=T>0\}$ и

$$
\Gamma(G)=\partial G \backslash \gamma(G) \equiv \gamma_{0}(G) \cup \Sigma(G)
$$

- параболическая граница области $G$ с боковой частью $\Sigma(G)=\Gamma(G) \backslash \gamma_{0}(G)$.

Рассмотрим на множестве $G \cup \gamma(G) \subset \mathbb{R}_{x, t}^{n+1}$ параболическую систему 2-го порядка из $m(m \geqslant 2)$ уравнений с "полным перемешиванием" вида

$$
\begin{aligned}
\mathscr{L}_{r}(u) \equiv & \sum_{k=1}^{m}\left(\sum_{i, j=1}^{n} a_{k, i j}^{(r)}(M) D_{i} D_{j} u_{k}+\sum_{i=1}^{n} b_{k, i}^{(r)}(M) D_{i} u_{k}\right. \\
& \left.+c_{k}^{(r)}(M) u_{k}-D_{t} u_{k}\right)=\Phi_{r}(M), \quad r=\overline{1, m}
\end{aligned}
$$

где при $M=(x, t) \equiv\left(x_{1}, \ldots, x_{n} ; t\right)$ действительные функции $a_{k, i j}^{(r)}, b_{k, i}^{(r)}, c_{k}^{(r)}$ принадлежат $C(G \cup \gamma(G)), r, k=\overline{1, m}, i, j=\overline{1, n}$, причем матрища

$$
a_{k}^{(r)}(M)=\left[a_{k, i j}^{(r)}(M)\right] \in M_{n \times n}, \quad r, k=\overline{1, m}
$$

предполагается симметрической и неотрицательно определенной на множестве $G \cup \gamma(G)$, так что ее собственные значения

$$
\begin{gathered}
0 \leqslant \lambda_{k, 1}^{(r)}(M) \leqslant \cdots \leqslant \lambda_{k, n}^{(r)}(M)<+\infty \\
\forall M=(x, t) \in G \cup \gamma(G), \quad r, k=\overline{1, m},
\end{gathered}
$$

неотрищательны.

Введем матрицы

$$
\begin{aligned}
A_{i j}(M) & =\left[a_{k, i j}^{(r)}(M)\right], \quad B_{i}(M)=\left[b_{k, i}^{(r)}(M)\right], \\
C(M) & =\left[c_{k}^{(r)}(M)\right], \quad I_{m}(M)=\left[\begin{array}{lll}
1 & & 0 \\
& \ddots & \\
0 & & 1
\end{array}\right] \in M_{m \times m}, \quad i, j=\overline{1, n},
\end{aligned}
$$

и векторы

$$
\begin{gathered}
u=\left[\begin{array}{c}
u_{1} \\
\ldots \\
u_{m}
\end{array}\right], \quad D_{i} u=\left[\begin{array}{c}
D_{i} u_{1} \\
\ldots \\
D_{i} u_{m}
\end{array}\right], \quad D_{i} D_{j} u=\left[\begin{array}{c}
D_{i} D_{j} u_{1} \\
\ldots \ldots \\
D_{i} D_{j} u_{m}
\end{array}\right], \\
D_{t} u \equiv I_{m}(M) D_{t} u=\left[\begin{array}{c}
D_{t} u_{1} \\
\ldots . \\
D_{t} u_{m}
\end{array}\right], \quad \Phi(M)=\left[\begin{array}{c}
\Phi_{1}(M) \\
\ldots \ldots \\
\ldots \\
\Phi_{m}(M)
\end{array}\right] \in M_{m \times 1} .
\end{gathered}
$$


Тогда параболическую систему 2-го порядка (1) можно записать в векторно-матричном виде

$$
\begin{aligned}
\mathscr{L}(u) \equiv & \sum_{i, j=1}^{n} A_{i j}(M) D_{i} D_{j} u+\sum_{i=1}^{n} B_{i}(M) D_{i} u \\
& +C(M) u-I_{m}(M) D_{t} u=\Phi(M) \quad \forall M \in G \cup \gamma(G) .
\end{aligned}
$$

Рассмотрим фиксированные векторы

$$
\begin{gathered}
p=\left[\begin{array}{c}
s_{1} \\
\ldots \\
s_{m}
\end{array}\right]>0, \quad f_{1}=\left[\begin{array}{c}
1 \\
\ldots \\
1
\end{array}\right], \quad f_{2}=\left[\begin{array}{c}
-s_{2} \\
s_{1} \\
0 \\
\ldots \\
0
\end{array}\right], \quad f_{3}=\left[\begin{array}{c}
0 \\
-s_{3} \\
s_{2} \\
0 \\
\ldots \\
0
\end{array}\right], \\
f_{l+1}=\left[\begin{array}{c}
0 \\
\ldots \\
0 \\
-s_{l+1} \\
s_{l} \\
0 \\
\ldots \\
0
\end{array}\right] \in M_{m \times 1}, \quad l=\overline{1, m-1},
\end{gathered}
$$

где $s_{k}>0, k=\overline{1, m}$, и $\sum_{k=1}^{m} s_{k}=1$ (так что (см. [10]) вектор $p>0$ положительный и стохастический). Введем матрищу

$$
E_{1}=f_{1} p^{T}=\left[\begin{array}{cccc}
s_{1} & s_{2} & \ldots & s_{m} \\
\ldots & \ldots & \ldots & \ldots \\
s_{1} & s_{2} & \ldots & s_{m}
\end{array}\right]=\left[\begin{array}{llll}
s_{1} f_{1} & s_{2} f_{1} & \ldots & s_{m} f_{1}
\end{array}\right] \in M_{m \times m}
$$

для которой в силу (5) имеем

$$
\begin{array}{lll}
E_{1} f_{1}=\lambda_{1} f_{1} & \text { при } & \lambda_{1}=1, \\
E_{1} f_{k}=\lambda_{k} f_{k} & \text { при } & \lambda_{k}=0, \quad k=\overline{2, m},
\end{array}
$$

так что матрица простой структуры $E_{1}$ из (6) подобна диагональной матрице

$$
F_{1}=\left[e_{1} \theta_{2} \ldots \theta_{m}\right] \in M_{m \times m},
$$

где $\theta_{i}=\left[\begin{array}{c}0 \\ \ldots \\ 0\end{array}\right]-i$-й столбец нулевой матрищы $[\theta]_{m}=\left[\theta_{1} \ldots \theta_{m}\right] \in M_{m \times m}$ и $e_{i}=\left[\delta_{k i}\right]-i$-й столбец единичной матрищы $I_{m}=\left[e_{1} \ldots e_{m}\right] \in M_{m \times m}$. Для вектора $e_{i}(i=\overline{1, m})$ имеем

$$
F_{1} e_{i}=\lambda_{i} e_{i}, \quad \lambda_{1}=1, \quad \lambda_{i}=0, \quad i=\overline{2, m},
$$


так что матрица $E_{1}$ из (6) имеет собственные векторы $f_{k}$ из $(5)$, соответствующие собственным значениям $\lambda_{k}$ из $(7)$, а матрица $F_{1}$ имеет собственные векторы $e_{i}$ из (9), соответствующие собственным значениям $\lambda_{i}$ из (9). Для матрицы $S_{m} \in M_{m \times m}$ (см. [5, с. 63]) перехода от $E_{1}$ к $F_{1}=S_{m}^{-1} E_{1} S_{m}$ имеем (см. (5)) представление

$$
S_{m}=\left[f_{1} f_{2} \ldots f_{m}\right] \in M_{m \times m},
$$

где в силу (9), (5) имеем

$$
S_{m} e_{k}=f_{k}, \quad S_{m}^{-1} f_{k}=e_{k}, \quad k=\overline{1, m},
$$

так что матрица $S_{m}$ переводит канонический базис $\left\{e_{1}, \ldots, e_{m}\right\}$ из $L_{e_{1} \ldots e_{m}}^{m} \equiv \mathbb{R}^{m}$ в базис $\left\{f_{1}, \ldots, f_{m}\right\}(5)$ (состоящий из собственных векторов матрищы $\left.E_{1}\right)$ из пространства $L_{f_{1} \ldots f_{m}}^{m}$, причем

$$
F_{1}=S_{m}^{-1} E_{1} S_{m}, \quad E_{1}=S_{m} F_{1} S_{m}^{-1} .
$$

Рассмотрим совокупность всех матриц из $M_{m \times m}$, образуюшую линейное пространство $L_{F_{1} \ldots F_{m^{2}}}^{m^{2}}$ с базисом $\left\{F_{1}, F_{2}, \ldots, F_{m^{2}}\right\}$, где

$$
\begin{gathered}
F_{l m+k}=\left[\theta_{1} \ldots \theta_{k-1} e_{l+1, k} \theta_{k+1} \ldots \theta_{m}\right] \in M_{m \times m} \\
l=\overline{0, m-1}, \quad k=\overline{1, m} .
\end{gathered}
$$

Здесь вектор-столбец $\theta_{i} \in M_{m \times 1}$ взят из (8), кроме того,

$$
e_{l+1, k}=\left[\begin{array}{c}
0 \\
\cdots \\
0 \\
1 \\
0 \\
\cdots \\
0
\end{array}\right]_{k}^{l+1} \in M_{m \times 1}, \quad l=\overline{0, m-1}, \quad k=\overline{1, m}
$$

Отметим, что матрица

$$
F_{l m+l+1}=\left[\theta_{1} \ldots \theta_{l} e_{l+1, l+1} \theta_{l+2} \ldots \theta_{m}\right] \in M_{m \times m}
$$

диагональная при $l=\overline{0, m-1}$, причем

$$
\sum_{l=0}^{m-1} F_{l m+l+1} \equiv I_{m} \in M_{m \times m}
$$


а матрица $F_{k}$ нильпотентная при $k \neq l m+l+1, l=\overline{0, m-1}$ (см. [10]), поскольку при $k \neq l+1$

$$
F_{l m+k} F_{l m+k}=[\theta]_{m}=\left[\theta_{1} \ldots \theta_{m}\right] \in M_{m \times m}
$$

Имеет место следующая таблица умножения:

$$
\begin{aligned}
F_{l m+p} F_{(p-1) m+k} & =F_{(p-1) m+k} & & \text { при } p, k=\overline{1, m}, \quad l=\overline{0, m-1}, \\
F_{q} F_{r} & =[\theta]_{m} & & \text { для остальных произведений. }
\end{aligned}
$$

Из $(10)$, учитьвая, что $s_{k} \neq 0(k=\overline{1, m})$, имеем

$$
\Delta_{m}=\operatorname{det} S_{m}= \begin{cases}1 & \text { при } m=2, \\ s_{2} s_{3} \ldots s_{m-1} & \text { при } m \geqslant 3,\end{cases}
$$

и тогда

$$
S_{m}^{-1}=\frac{1}{\Delta_{m}}\left[A_{i j}\right]^{T}=\frac{1}{\Delta_{m}}\left[\begin{array}{cccc}
A_{11} & A_{21} & \ldots & A_{m 1} \\
\ldots \ldots & \ldots \ldots & \ldots & \ldots \\
A_{1 m} & A_{2 m} & \ldots & A_{m m}
\end{array}\right]
$$

где $A_{i j}$ - алгебраическое дополнение элемента $a_{i j}$ матрищы $S_{m}=\left[a_{i j}\right]=$ $=\left[f_{1} f_{2} \ldots f_{m}\right] \in M_{m \times m}$ из $(10)$.

ЗАМЕЧАНИЕ 1 . При $m=2$ имеем

$$
S_{2}=\left[f_{1} f_{2}\right]=\left[\begin{array}{cc}
1 & -s_{2} \\
1 & s_{1}
\end{array}\right], \quad \Delta_{2}=1, \quad S_{2}^{-1}=\left[\begin{array}{cc}
A_{11} & A_{21} \\
A_{12} & A_{22}
\end{array}\right]=\left[\begin{array}{cc}
s_{1} & s_{2} \\
-1 & 1
\end{array}\right]
$$

т.е. $A_{11}=s_{1}, A_{12}=-1, A_{21}=s_{2}, A_{22}=1$.

При $m=3$ имеем

$$
\begin{gathered}
S_{3}=\left[f_{1} f_{2} f_{3}\right]=\left[\begin{array}{ccc}
1 & -s_{2} & 0 \\
1 & s_{1} & -s_{3} \\
1 & 0 & s_{2}
\end{array}\right], \\
\Delta_{3}=s_{2} \neq 0, \quad S_{3}^{-1}=\frac{1}{s_{2}}\left[\begin{array}{ccc}
A_{11} & A_{21} & A_{31} \\
\ldots \ldots & \ldots \ldots \\
A_{31} & A_{32} & A_{33}
\end{array}\right],
\end{gathered}
$$

где $A_{11}=s_{1} s_{2}, A_{21}=s_{2}^{2}, A_{31}=s_{2} s_{3}, A_{12}=-\left(s_{1}+s_{3}\right), A_{22}=s_{2}, A_{32}=s_{3}$, $A_{13}=-s_{1}, A_{23}=-s_{2}, A_{33}=s_{1}+s_{2}$.

Введем представления

$$
E_{k}=S_{m} F_{k} S_{m}^{-1}, \quad k=\overline{1, m^{2}}
$$

Тогда имеем, учитьвая (11), (10) (см. (12), (5)),

$$
\begin{aligned}
S_{m} F_{l m+k} & =S_{m}\left[\theta_{1} \ldots \theta_{k-1} e_{l+1, k} \theta_{k+1} \ldots \theta_{m}\right] \\
& =\left[\theta_{1} \ldots \theta_{k-1} f_{l+1} \theta_{k+1} \ldots \theta_{m}\right], \quad l=\overline{0, m-1}, \quad k=\overline{1, m} .
\end{aligned}
$$


Учитывая (19), (20), имеем при $m \geqslant 2$

$$
\begin{aligned}
E_{1} & =f_{1} p^{T}=\left[s_{1} f_{1} s_{2} f_{1} \ldots s_{m} f_{1}\right]=\left(S_{m} F_{1}\right) S_{m}^{-1} \\
& =\frac{1}{\Delta_{m}}\left[f_{1} \theta_{2} \ldots \theta_{m}\right]\left[A_{i j}\right]^{T}=\frac{1}{\Delta_{m}}\left[A_{11} f_{1} A_{21} f_{1} \ldots A_{m 1} f_{1}\right],
\end{aligned}
$$

так что

$$
\begin{aligned}
A_{11}=s_{1} s_{2} \ldots s_{m-1}, \quad A_{21} & =s_{2}^{2} s_{3} \ldots s_{m-1}, \quad \ldots, \quad A_{m 1}=s_{2} \ldots s_{m-1} s_{m} \\
\frac{1}{\Delta_{m}} A_{i 1} & =s_{i}, \quad i=\overline{1, m}, \quad \forall m \geqslant 2
\end{aligned}
$$

и при $k=\overline{1, m}, l=0$

$$
\begin{aligned}
E_{k} & =S_{m} F_{k} S_{m}^{-1}=\left[\theta_{1} \ldots \theta_{k-1} f_{1} \theta_{k+1} \ldots \theta_{m}\right] S_{m}^{-1} \\
& \equiv \frac{1}{\Delta_{m}}\left[\theta_{1} \ldots \theta_{k-1} f_{1} \theta_{k+1} \ldots \theta_{m}\right]\left[A_{i j}\right]^{T} \\
& =\frac{1}{\Delta_{m}}\left[A_{1 k} f_{1} A_{2 k} f_{1} \ldots A_{m k} f_{1}\right], \\
E_{m+k} & =S_{m} F_{m+k} S_{m}^{-1}=\frac{1}{\Delta_{m}}\left[\theta_{1} \ldots \theta_{k-1} f_{2} \theta_{k+1} \ldots \theta_{m}\right]\left[A_{i j}\right]^{T} \\
& =\frac{1}{\Delta_{m}}\left[A_{1 k} f_{2} A_{2 k} f_{2} \ldots A_{m k} f_{2}\right], \quad k=\overline{1, m}, \\
E_{l m+k} & =S_{m} F_{l m+k} S_{m}^{-1}=\left[\theta_{1} \ldots \theta_{k-1} f_{l+1} \theta_{k+1} \ldots \theta_{m}\right] S_{m}^{-1} \\
& =\frac{1}{\Delta_{m}}\left[A_{1 k} f_{l+1} A_{2 k} f_{l+1} \ldots A_{m k} f_{l+1}\right], \quad l=\overline{2, m-1}, \quad k=\overline{1, m} .
\end{aligned}
$$

ЗАМЕЧАНИЕ 2 . При $m=2$

$$
f_{1}=\left[\begin{array}{l}
1 \\
1
\end{array}\right], \quad f_{2}=\left[\begin{array}{c}
-s_{2} \\
s_{1}
\end{array}\right], \quad \Delta_{2}=1, \quad s_{1}+s_{2}=1
$$

и поэтому

$$
\begin{array}{ll}
E_{1}=\left[\begin{array}{ll}
s_{1} f_{1} & s_{2} f_{1}
\end{array}\right], & E_{2}=\left[\begin{array}{ll}
-f_{1} & f_{1}
\end{array}\right], \\
E_{3}=\left[\begin{array}{ll}
s_{1} f_{2} & s_{2} f_{2}
\end{array}\right], & E_{4}=\left[\begin{array}{ll}
-f_{2} & f_{2}
\end{array}\right] .
\end{array}
$$

При $m=3$

$$
f_{1}=\left[\begin{array}{l}
1 \\
1 \\
1
\end{array}\right], \quad f_{2}=\left[\begin{array}{c}
-s_{2} \\
s_{1} \\
0
\end{array}\right], \quad f_{3}=\left[\begin{array}{c}
0 \\
-s_{3} \\
s_{2}
\end{array}\right], \quad \Delta_{3}=s_{2}, \quad s_{1}+s_{2}+s_{3}=1
$$


и поэтому

$$
\begin{array}{rlrl}
E_{1} & =\left[\begin{array}{lll}
s_{1} f_{1} & s_{2} f_{1} & s_{3} f_{1}
\end{array}\right], & E_{2}=\frac{1}{s_{2}}\left[\begin{array}{lll}
\left.s_{2}+s_{3}\right) f_{1} & s_{2} f_{1} & s_{3} f_{1}
\end{array}\right], \\
E_{3}=\frac{1}{s_{2}}\left[\begin{array}{llll}
-s_{1} f_{1} & -s_{2} f_{1} & \left(s_{1}+s_{2}\right) f_{1}
\end{array}\right], & E_{4}=\frac{1}{s_{2}}\left[\begin{array}{lll}
s_{1} s_{2} f_{2} & s_{2}^{2} f_{2} & s_{2} s_{3} f_{2}
\end{array}\right], \\
E_{5}=\frac{1}{s_{2}}\left[\begin{array}{llll}
-\left(s_{2}+s_{3}\right) f_{2} & s_{2} f_{2} & s_{3} f_{2}
\end{array}\right], & E_{6}=\frac{1}{s_{2}}\left[\begin{array}{lll}
-\left(s_{2}+s_{3}\right) f_{3} & s_{2} f_{3} & s_{3} f_{3}
\end{array}\right], \\
E_{7}=\frac{1}{s_{2}}\left[\begin{array}{lllll}
s_{1} s_{2} f_{3} & s_{2}^{2} f_{3} & s_{2} s_{3} f_{3}
\end{array}\right], & E_{8}=\frac{1}{s_{2}}\left[\begin{array}{lll}
-\left(s_{2}+s_{3}\right) f_{3} & s_{2} f_{3} & s_{3} f_{3}
\end{array}\right], \\
E_{9}=\frac{1}{s_{2}}\left[\begin{array}{lll}
-s_{1} f_{3} & -s_{2} f_{3} & \left.\left(s_{1}+s_{2}\right) f_{3}\right] .
\end{array}\right.
\end{array}
$$

Поскольку в силу (19)

$$
E_{k} E_{l}=S_{m} F_{k} S_{m}^{-1} S_{m} F_{l} S_{m}^{-1} \equiv S_{m} F_{k} F_{l} S_{m}^{-1}
$$

то из таблищы умножения (16) для матриц $F_{k}$ получаем таблицу умножения для матриц $E_{k}$ :

$$
\begin{gathered}
E_{l m+p} E_{(p-1) m+k}=E_{(p-1) m+k}, \quad p, k=\overline{1, m}, \quad l=\overline{0, m-1}, \\
E_{q} E_{r}=[\theta]_{m} \in M_{m \times m}
\end{gathered}
$$

для остальных индексов $q$ и $r$. В частности, поскольку $E_{l m+l+1}=S_{m} F_{l m+l+1} S_{m}^{-1}$, то матрища $E_{l m+l+1}$ является матрицей простой структуры, подобной диагональной матрице $F_{l m+l+1}$ при $l=\overline{0, m-1}$, а матрица $E_{l m+k} \quad \forall l=\overline{0, m-1}, k \neq$ $l+1$, будет в силу (15) нильпотентной (как и матрища $F_{l m+k}$ при $l=\overline{0, m-1}$, $k \neq l+1)$. Поскольку $\left\{F_{1}, F_{2}, \ldots, F_{m^{2}}\right\}$ является базисом в $L^{m^{2}} \equiv L_{F_{1} \ldots F_{m^{2}}}^{m^{2}}$, то в силу (19) $E_{k} \sim F_{k}, k=\overline{1, m^{2}}$, и поэтому $\left\{E_{1}, E_{2}, \ldots, E_{m^{2}}\right\}$ будет базисом в $L^{m^{2}} \equiv L_{E_{1} \ldots E_{m^{2}}}^{m^{2}}$. Тогда имеем разложение

$$
L_{E_{1} \ldots E_{m^{2}}}^{m^{2}}=L_{E_{1}}^{m} E_{m+2} E_{2 m+3} \ldots E_{(m-1) m+m} \bigoplus_{\substack{r \neq l m+l+1 \\ l=\overline{0, m-1}}} L_{E_{r}}^{1}
$$

Докажем лемму о разложении матрищы из $M_{m \times m}$ в сумму матрищ простой структуры и нильпотентных матрищ по базису $\left\{E_{1}, \ldots, E_{m^{2}}\right\}$.

ЛЕмма 1. Пусть $E_{1}=\left[s_{1} f_{1} \ldots s_{m} f_{1}\right]$ uз (6), $S_{m}$ uз (10), $S_{m}^{-1} u_{3}(12)-$ матрици перехода от $L_{e_{1} \ldots e_{m}}^{m} \kappa L_{f_{1} \ldots f_{m}}^{m}$ u om $L_{f_{1} \ldots f_{m}}^{m} \kappa L_{e_{1} \ldots e_{m}}^{m}$ coответственно (см. (5) и (9)), причем $E_{k}\left(k=\overline{1, m^{2}}\right)$ взяты из (19), где матриць $E_{l m+l+1} \quad(l=\overline{0, m-1})$ простой структуры, а матрицы $E_{r}$ nри $r \neq l m+l+1$ $(l=\overline{0, m-1})$ нильпотентнь. 
Тогда для любой матрицы

$$
b=\left[b_{i j}\right] \in M_{m \times m}
$$

в базисе $\left\{E_{1}, \ldots, E_{m^{2}}\right\}$ имеет место представление

$$
b=\sum_{r=1}^{m^{2}} \beta_{r} E_{r} .
$$

При $m=2$ (см. (25) из замечания 2)

$$
b=\left[\begin{array}{ll}
b_{11} & b_{12} \\
b_{21} & b_{22}
\end{array}\right]=\sum_{r=1}^{4} \beta_{r} E_{r},
$$

причем

$$
\begin{aligned}
& \beta_{1}=s_{1}\left(b_{11}+b_{12}\right)+s_{2}\left(b_{21}+b_{22}\right), \\
& \beta_{2}=s_{1}^{2} b_{12}+s_{1} s_{2}\left(b_{22}-b_{11}\right)-s_{2}^{2} b_{21}, \\
& \beta_{3}=b_{22}+b_{21}-b_{11}-b_{12}, \\
& \beta_{4}=s_{1}\left(b_{22}-b_{12}\right)+s_{2}\left(b_{11}-b_{21}\right) .
\end{aligned}
$$

При $m=3$ (см. (26) замечания 2), $\Delta_{2}=s_{2} \neq 0$

$$
b=\left[\begin{array}{ccc}
b_{11} & b_{12} & b_{13} \\
\ldots & \ldots & \ldots \\
b_{31} & b_{32} & b_{33}
\end{array}\right]=\sum_{r=1}^{9} \beta_{r} E_{r}
$$

где

$$
\begin{gathered}
\beta_{1}=\sum_{k=1}^{3} s_{k} \sum_{r=1}^{3} b_{k r}, \quad \beta_{2}=\sum_{k=1}^{3} s_{k}\left(-s_{2} b_{k 1}+s_{1} b_{k 2}\right) \\
\beta_{3}=\sum_{k=1}^{3} s_{k}\left(-s_{3} b_{k 2}+s_{2} b_{k 3}\right), \quad s_{2} \beta_{4}=\sum_{k=1}^{3}\left(s_{k} \sum_{r=1}^{3} b_{k r}-b_{k 1}\right) \\
s_{2} \beta_{5}=\sum_{k=1}^{3} s_{k}\left(-s_{2} b_{k 1}+s_{1} b_{k 2}\right)+s_{2} b_{11}-s_{1} b_{12} \\
s_{2} \beta_{6}=\sum_{k=1}^{3} s_{k}\left(-s_{3} b_{k 2}+s_{2} b_{k 3}\right)+s_{3} b_{12}-s_{2} b_{13} \\
s_{2} \beta_{7}=-\sum_{k=1}^{3}\left(s_{k} \sum_{r=1}^{3} b_{k r}+b_{3 k}\right) \\
s_{2} \beta_{8}=\sum_{k=1}^{3} s_{k}\left(s_{2} b_{k 1}-s_{1} b_{k 2}\right)+s_{1} b_{32}-s_{2} b_{31} \\
s_{2} \beta_{9}=\sum_{k=1}^{3} s_{k}\left(s_{3} b_{k 2}-s_{2} b_{k 3}\right)+s_{2} b_{33}-s_{3} b_{32}
\end{gathered}
$$


При $m \geqslant 4$ (см. $(22)-(24))$ и $\beta_{l m+k}$ и при (см. (17))

$$
E_{l m+k}, \quad l=\overline{0, m-1}, \quad k=\overline{1, m}, \quad \Delta_{m}=s_{2} \ldots s_{m-1} \neq 0
$$

имеем разложение

$$
b=\sum_{l=0}^{m-1} \sum_{k=1}^{m} \beta_{l m+k} E_{l m+k}
$$

где

$$
\Delta_{m}\left[\begin{array}{ccc}
\beta_{1} & \ldots & \beta_{m} \\
\beta_{m+1} & \ldots & \beta_{m+m} \\
\ldots \ldots \ldots & \ldots & \ldots \ldots \\
\beta_{(m-1) m+1} & \ldots & \beta_{m^{2}}
\end{array}\right]=\left[\alpha_{i j}\right] S_{m}
$$

причем (см. (28), (18)) $\alpha_{i j}=\sum_{r=1}^{m} A_{r i} b_{r j}$ и (см. (5))

$$
\Delta_{m} \beta_{1}=\left[\alpha_{11} \alpha_{12} \ldots \alpha_{1 m}\right] f_{1}=\sum_{j=1}^{m} \alpha_{1 j}
$$

При $k=\overline{2, m}$

$$
\begin{aligned}
\Delta_{m} \beta_{k} & =\left[\alpha_{11} \alpha_{12} \ldots \alpha_{1 m}\right] f_{k}=-s_{k} \alpha_{1 k-1}+s_{k-1} \alpha_{1 k} \\
\Delta_{m} \beta_{l m+1} & =\left[\alpha_{l+11} \ldots \alpha_{l+1 m}\right] f_{1}=\sum_{j=1}^{m} \alpha_{l+1 j}, \quad l=\overline{1, m-1} .
\end{aligned}
$$

Наконец, при $k=\overline{2, m}(l=\overline{1, m-1})$ имеем

$$
\Delta_{m} \beta_{l m+k}=\left[\alpha_{l+11} \ldots \alpha_{l+1 m}\right] f_{k}=-s_{k} \alpha_{l+1 k-1}+s_{k-1} \alpha_{l+1 k} .
$$

ДоКАЗАТЕЛЬСТво ЛЕммЫ 1 . Рассмотрим при $m \geqslant 3$ любую матрицу $b \in$ $M_{m \times m}$ из (28) в старом базисе $\left\{e_{1}, e_{2}, \ldots, e_{m}\right\}$ из (9), которому при $m \geqslant 3$ соответствует (см. (10), (18)) матрица

$$
\begin{aligned}
b_{f} & =S_{m}^{-1} b S_{m}=\frac{1}{\Delta_{m}}\left[A_{i j}\right]^{T}\left[b_{i j}\right]\left[f_{1} \ldots f_{m}\right] \\
& \equiv \frac{1}{\Delta_{m}}\left[\begin{array}{ccc}
A_{11} & \ldots & A_{m 1} \\
\ldots \ldots & \ldots & \ldots \\
A_{1 m} & \ldots & A_{m m}
\end{array}\right]\left[\begin{array}{ccc}
b_{11} & \ldots & b_{1 m} \\
\ldots \ldots & \ldots & \ldots \\
b_{m 1} & \ldots & b_{m m}
\end{array}\right]\left[f_{1} \ldots f_{m}\right] .
\end{aligned}
$$

Разлагая $b_{f} \in M_{m \times m}$ по базису $\left\{F_{1}, F_{2}, \ldots, F_{m^{2}}\right\}$ в $L_{F_{1} \ldots F_{m^{2}}}^{m^{2}}$, имеем

$$
b_{f}=\sum_{k=1}^{m^{2}} \beta_{k} F_{k} \equiv\left(S_{m}^{-1} b\right) S_{m}=\left[\begin{array}{ccc}
\beta_{1} & \ldots & \beta_{m} \\
\beta_{m+1} & \ldots & \beta_{m+m} \\
\ldots \ldots \ldots \ldots & \ldots & \ldots \\
\beta_{(m-1) m+1} & \ldots & \beta_{m^{2}}
\end{array}\right] \text {, }
$$


и поскольку

$$
S_{m}^{-1} b=\frac{1}{\Delta_{m}}\left[A_{i j}\right]^{T}\left[b_{i j}\right] \equiv \frac{1}{\Delta_{m}}\left[\alpha_{i j}\right]
$$

где $\left[\alpha_{i j}\right]=\left[A_{i j}\right]^{T}\left[b_{i j}\right]$, то

$$
\alpha_{i j}=\sum_{r=1}^{m} A_{r i} b_{r j}, \quad i, j=\overline{1, m}
$$

откуда с учетом (33) имеем

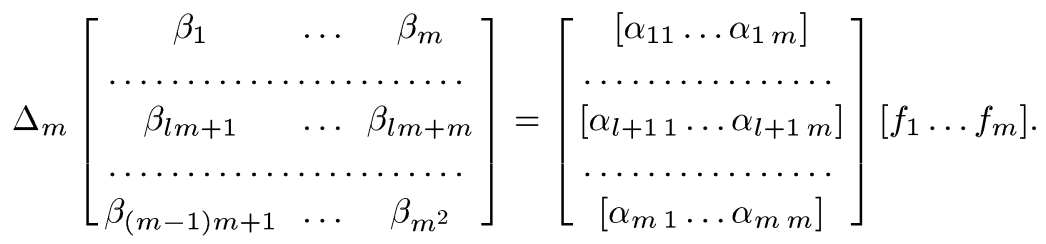

Отсюда при $l=\overline{0, m-1}, k=\overline{1, m}$ имеем для $m \geqslant 3$

$$
\Delta_{m} \beta_{l m+k}=\left[\alpha_{l+11} \ldots \alpha_{l+1 m}\right] f_{k}
$$

т.e.

$$
\begin{aligned}
& \Delta_{m} \beta_{l m+1}=\sum_{j=1}^{m} \alpha_{l+1 j} \quad \text { при } \quad k=1, \\
& \Delta_{m} \beta_{l m+k}=-s_{k} \alpha_{l+1 k-1}+s_{k-1} \alpha_{l+1 k} \quad \text { при } \quad k=\overline{2, m}, \quad l=\overline{0, m-1} .
\end{aligned}
$$

Но тогда в силу (19) из (33) имеем представление

$$
\begin{aligned}
b & =S_{m} b_{f} S_{m}^{-1}=\sum_{r=1}^{m^{2}} \beta_{r} S_{m} F_{r} S_{m}^{-1} \\
& \equiv \sum_{r=1}^{m^{2}} \beta_{r} E_{r} \equiv \sum_{l=0}^{m-1} \sum_{k=1}^{m} \beta_{l m+k} E_{l m+k}
\end{aligned}
$$

что и завершает ввиду $(34),(35)$ доказательство разложения $(32)$ при $m \geqslant 3$.

В случае $m=2$ доказательство (30) проводится аналогично.

Докажем лемму о сведении векторно-матричной алгебраической системы из $m$ уравнений к $m$ скалярным уравнениям. 
ЛЕмма 2. Пусть

$$
b u=h,
$$

әде при $m \geqslant 2$ с учетом (28)

$$
b \in M_{m \times m}, \quad u=\left[\begin{array}{c}
u_{1} \\
\ldots \\
u_{m}
\end{array}\right], \quad h=\left[\begin{array}{c}
h_{1} \\
\ldots \\
h_{m}
\end{array}\right] \in M_{m \times 1}
$$

и имеет место представление (29) из леммы 1.

Тогда в случае $m=2$ при

$$
v_{1}=s_{1} u_{1}+s_{2} u_{2}, \quad v_{2}=u_{2}-u_{1}, \quad H_{1}=s_{1} h_{1}+s_{2} h_{2}, \quad H_{2}=h_{2}-h_{1}
$$

имеем (см. (30) и (5))

$$
\left(\beta_{1} v_{1}+\beta_{2} v_{2}\right) f_{1}+\left(\beta_{3} v_{1}+\beta_{4} v_{2}\right) f_{2}=H_{1} f_{1}+H_{2} f_{2},
$$

что дает два скалярных уравнения для $v_{1}$ u $v_{2}$ :

$$
\beta_{1} v_{1}+\beta_{2} v_{2}=H_{1}, \quad \beta_{3} v_{1}+\beta_{4} v_{2}=H_{2},
$$

где $\beta_{r} \quad(r=\overline{1,4})$ взяты из (30).

B случае $m=3$ nри

$$
\begin{aligned}
& v_{1}=\sum_{k=1}^{3} s_{k} u_{k}, \quad v_{2}=\frac{1}{s_{2}}\left(\sum_{k=1}^{3} s_{k} u_{k}-u_{1}\right), \quad v_{3}=\frac{1}{s_{2}}\left(u_{3}-\sum_{k=1}^{3} s_{k} u_{k}\right), \\
& H_{1}=\sum_{k=1}^{3} s_{k} h_{k}, \quad H_{2}=\frac{1}{s_{2}}\left(\sum_{k=1}^{3} s_{k} h_{k}-h_{1}\right), \quad H_{3}=\frac{1}{s_{2}}\left(h_{3}-\sum_{k=1}^{3} s_{k} h_{k}\right), \\
& \Delta_{3}=s_{2} \neq 0
\end{aligned}
$$

имеем (см. (31))

$$
\left(\sum_{k=1}^{3} \beta_{k} v_{k}\right) f_{1}+\left(\sum_{k=1}^{3} \beta_{k+3} v_{k}\right) f_{2}+\left(\sum_{k=1}^{3} \beta_{k+6} v_{k}\right) f_{3}=\sum_{k=1}^{3} H_{k} f_{k}
$$

что дает три скалярных уравнения для $v_{k} \quad(k=\overline{1,3})$ :

$$
\sum_{k=1}^{3} \beta_{k} v_{k}=H_{1}, \quad \sum_{k=1}^{3} \beta_{k+3} v_{k}=H_{2}, \quad \sum_{k=1}^{3} \beta_{k+6} v_{k}=H_{3}
$$

причем $\beta_{r} \quad(r=\overline{1,9})$ взяты из (31) леммы 1. 
Наконеи, при $m \geqslant 4, \Delta_{m}=s_{2} \ldots s_{m-1} \neq 0$, учитывая (18), при

$$
\begin{aligned}
v_{k} & =\frac{1}{\Delta_{m}} \sum_{i=1}^{m} A_{i k} u_{i}, & k & =\overline{1, m}, \\
H_{l+1} & =\frac{1}{\Delta_{m}} \sum_{i=1}^{m} A_{i l+1} h_{i}, & l & =\overline{0, m-1},
\end{aligned}
$$

имеем (см. (31))

$$
\sum_{l=0}^{m-1}\left(\sum_{k=1}^{m} \beta_{l m+k} v_{k}\right) f_{l+1}=\sum_{l=0}^{m-1} H_{l+1} f_{l+1}
$$

где $\beta_{r} \quad\left(r=\overline{1, m^{2}}\right)$ взяты из (32) леммы 1 , что дает $m$ скалярных уравнений для $v_{k} \quad(k=\overline{1, m})$ из $(39)$ :

$$
\sum_{k=1}^{m} \beta_{k} v_{k}=H_{1}, \quad \sum_{k=1}^{m} \beta_{k+m} v_{k}=H_{2}, \quad \ldots, \quad \sum_{k=1}^{m} \beta_{k+m(m-1)} v_{k}=H_{m}
$$

ДоКАЗАТЕЛЬСТво. Поскольку в силу (14), (19)

$$
S_{m}\left(\sum_{l=0}^{m-1} F_{m l+l+1}\right) S_{m}^{-1} \equiv I_{m}=\left[e_{1} \ldots e_{m}\right] \in M_{m \times m}
$$

то

$$
\sum_{l=0}^{m-1} E_{m l+l+1}=S_{m}\left(\sum_{l=0}^{m-1} F_{m l+l+1}\right) S_{m}^{-1} \equiv I_{m} \in M_{m \times m},
$$

откуда $\forall b \in M_{m \times m}$ из (28), используя представление (32) леммы 1 (при $m \geqslant 3$ ), имеем представление

$$
b=\sum_{l=0}^{m-1} \sum_{k=1}^{m} \beta_{l m+k} E_{l m+k} \equiv \sum_{r=1}^{m^{2}} \beta_{r} F_{r}
$$

дающее

$$
b \equiv I_{m} b \equiv \sum_{l=0}^{m-1} E_{m l+l+1} \sum_{r=1}^{m} \beta_{r} E_{r} .
$$

Используя таблицу умножения (27) для матриц $E_{r} \quad\left(r=\overline{1, m^{2}}\right)$, имеем $E_{l m+l+1} E_{r}=E_{l m+k}$ лишь при $r=l m+k$, так что из (42) получаем

$$
\sum_{r=1}^{m^{2}} \sum_{l=0}^{m-1} \beta_{r} E_{m l+l+1} E_{r}=\sum_{k=1}^{m} \sum_{l=0}^{m-1} \beta_{l m+k} E_{l m+k}
$$


в силу того, что возможно лишь $r=(p-1) m+k$ при $p, k=\overline{1, m}$, что дает представление

$$
b=\sum_{k=1}^{m} \sum_{l=0}^{m-1} \beta_{l m+k} E_{l m+k} .
$$

Тем самым система (36) приведена к системе

$$
\sum_{k=1}^{m} \sum_{l=0}^{m-1} \beta_{l m+k} E_{l m+k} u=\sum_{l=0}^{m-1} E_{m l+l+1} u,
$$

где в силу (22)-(24) имеем при $l=\overline{0, m-1}, k=\overline{1, m}$

$$
E_{l m+k} u=\frac{1}{\Delta_{m}}\left[A_{1 k} f_{l+1} \ldots A_{m k} f_{l+1}\right] u \equiv v_{k} f_{l+1} .
$$

Здесь

$$
\begin{gathered}
v_{k}=\frac{1}{\Delta_{m}} \sum_{i=1}^{m} A_{i k} u_{i}, \\
E_{l m+l+1} h=\frac{1}{\Delta_{m}}\left[A_{1 l+1} f_{l+1} \ldots A_{m l+1} f_{l+1}\right] h \equiv H_{l+1} f_{l+1}
\end{gathered}
$$

причем

$$
H_{l+1}=\frac{1}{\Delta_{m}} \sum_{i=1}^{m} A_{i l+1} h_{i}, \quad l=\overline{0, m-1} .
$$

Итак, система (36) приведена к векторной системе (40), которую можно записать как систему $m$ скалярных уравнений (41) для $v_{k}(k=\overline{1, m})$ из (39). Доказательство леммы 2 в случае $m=2$ проводится аналогично.

ОПРЕДЕЛЕнИЕ 1 . Матрица $b=\left[b_{i j}\right] \in M_{m \times m}$ из (28), для которой имеет место представление (29) (т.е. (30) при $m=2,(31)$ при $m=3$ и (32) при $m \geqslant 4$ ) леммы 1 , удовлетворяет условию (А. $k$ ), где $k=\overline{1, m}$, если в (41)

$$
\beta_{(k-1) m+i}=0 \quad \forall i=\overline{1, k-1}, \overline{k+1, m} \quad(\forall i \neq k) .
$$

ЗАмЕчАнИЕ 3 . При выполнении условия (А.1) для $b \in M_{2 \times 2}$ в случае $m=2$ имеем из (30)

$$
\begin{aligned}
& \beta_{1}=s_{1}\left(b_{11}+b_{12}\right)+s_{2}\left(b_{21}+b_{22}\right) \\
& \beta_{2} \equiv s_{1}^{2} b_{12}+s_{1} s_{2}\left(b_{22}-b_{11}\right)-s_{2}^{2} b_{21}=0
\end{aligned}
$$

так что при $s_{1} \neq 0$

$$
\beta_{1}=\frac{1}{s_{1}} \sum_{r=1}^{2} s_{r} b_{r 1}
$$


При выполнении условия (А.1) для $b \in M_{3 \times 3}$ в случае $m=3$ имеем из (31)

$$
\begin{aligned}
& \beta_{1}=\sum_{k=1}^{3} s_{k} \sum_{r=1}^{3} b_{k r} \\
& \beta_{2} \equiv \sum_{k=1}^{3}\left(-s_{2} b_{k 1}+s_{1} b_{k 2}\right)=0 \\
& \beta_{3} \equiv \sum_{k=1}^{3} s_{k}\left(-s_{3} b_{k 2}+s_{2} b_{k 3}\right)=0,
\end{aligned}
$$

так что

$$
\beta_{1}=\frac{1}{s_{1}} \sum_{r=1}^{m} s_{r} b_{r 1}
$$

При вьполнении условия (А.2) для $b \in M_{3 \times 3}$ в случае $m=3$ имеем из $(31)\left(s_{2} \neq 0\right)$

$$
\begin{gathered}
s_{2} \beta_{5}=-s_{2} \sum_{k=1}^{3} s_{k} b_{k 1}+s_{1} \sum_{k=1}^{3} s_{k} b_{k 2}+s_{2} b_{11}-s_{1} b_{12}, \\
s_{2} \beta_{4}=0, \quad s_{2} \beta_{6}=0,
\end{gathered}
$$

что дает

$$
s_{2} \beta_{5}=b_{12}-\sum_{k=1}^{3} s_{k} b_{k 2},
$$

так что при выполнении (А.1) и (А.2) одновременно для $b \in M_{3 \times 3}$ в случае $m=3$ имеем

$$
\beta_{1}=\frac{1}{s_{1}} \sum_{r=1}^{3} s_{r} b_{r 1}, \quad s_{2} \beta_{5}=b_{12}-\sum_{k=1}^{3} s_{k} b_{k 2} .
$$

Наконец, при выполнении условия (А.1) для $b \in M_{m \times m}$ в случае $m \geqslant 4$, учитывая, что в силу (21)

$$
\frac{1}{\Delta_{m}} \alpha_{1 j}=\frac{1}{\Delta_{m}} \sum_{r=1}^{m} A_{r 1} b_{r j} \equiv \sum_{r=1}^{m} s_{r} b_{r j},
$$

при $k=\overline{2, m}$ из $(32)$ имеем

$$
\beta_{k} \equiv \frac{1}{\Delta_{m}}\left(-s_{k} \alpha_{1 k-1}+s_{k-1} \alpha_{1 k}\right)=0
$$

учитывая соотношения

$$
\frac{1}{\Delta_{m}} \alpha_{1 k-1}=\sum_{r=1}^{m} s_{r} b_{r k-1}, \quad \frac{1}{\Delta_{m}} \alpha_{1 k}=\sum_{r=1}^{m} s_{r} b_{r k},
$$


получаем

$$
s_{k} \sum_{r=1}^{m} s_{r} b_{r k-1}=s_{k-1} \sum_{r=1}^{m} s_{r} b_{r k}
$$

так что

$$
\beta_{1}=\sum_{r=1}^{m} s_{r} b_{r 1}+\sum_{k=2}^{m} \sum_{r=1}^{m} s_{r} b_{r k}
$$

Но при $k=\overline{2, m}$

$$
\begin{aligned}
\sum_{r=1}^{m} s_{r} b_{r k} & =\frac{s_{k}}{s_{k-1}} \sum_{r=1}^{m} s_{r} b_{r k-1} \\
& =\frac{s_{k}}{s_{k-1}} \frac{s_{k-1}}{s_{k-2}} \cdots \frac{s_{2}}{s_{1}} \sum_{r=1}^{m} s_{r} b_{r 1} \equiv \frac{s_{k}}{s_{1}} \sum_{r=1}^{m} s_{r} b_{r} 1
\end{aligned}
$$

что дает при выполнении условия (А.1)

$$
\beta_{1}=\left(1+\sum_{k=2}^{m} \frac{s_{k}}{s_{1}}\right) \sum_{r=1}^{m} s_{r} b_{r 1} \equiv \frac{1}{s_{1}}\left(\sum_{k=1}^{m} s_{k}\right) \sum_{r=1}^{m} s_{r} b_{r 1} .
$$

А поскольку в силу стохастичности вектора $p>0$ из (5) $\sum_{k=1}^{m} s_{k}=1$, то

$$
\beta_{1}=\frac{1}{s_{1}} \sum_{r=1}^{m} s_{r} b_{r 1} \quad \forall m \geqslant 2
$$

Рассмотрим теперь матрищы (2) системы (4), определенные на множестве $G \cup \gamma(G) \subset \mathbb{R}_{x, t}^{n+1}$, и введем для них представления (32) из леммы 1 с одними и теми же фиксированными векторами $p, f_{k} \in M_{m \times 1}(k=\overline{1, m})$ из $(5)$. Тогда имеем при $i, j=\overline{1, n}$

$$
\begin{gathered}
A_{i j}(M)=\left[a_{k, i j}^{(r)}(M)\right] \equiv \sum_{l=0}^{m-1} \sum_{k=1}^{m} \beta_{l m+k, i j}(M) E_{l m+k} \\
B_{i}(M)=\left[b_{k, i}^{(r)}(M)\right] \equiv \sum_{l=0}^{m-1} \sum_{k=1}^{m} \beta_{l m+k, i}(M) E_{l m+k} \\
C(M)=\left[c_{k}^{(r)}(M)\right] \equiv \sum_{l=0}^{m-1} \sum_{k=1}^{m} \beta_{l m+k}(M) E_{l m+k} .
\end{gathered}
$$

Пусть матрищы (47)-(49)удовлетворяют условию (А.1) определения 1. Тогда в силу (40) леммы 2 и (46) замечания 3 получаем, в частности, скалярное уравнение 2-го порядка для

$$
v_{1} \equiv \sum_{k=1}^{m} s_{k} u_{k} \in C_{x, t}^{(2,1)}(G \cup \gamma(G))
$$


при $H_{1}(M) \equiv \sum_{k=1}^{m} s_{k} \Phi_{k}(M)$ :

$$
\begin{aligned}
L_{1} v_{1} \equiv & \frac{1}{s_{1}} \sum_{i, j=1}^{n}\left(\sum_{r=1}^{m} s_{r} a_{1, i j}^{(r)}(M)\right) D_{i} D_{j} v_{1} \\
& +\frac{1}{s_{1}} \sum_{i=1}^{n}\left(\sum_{r=1}^{m} s_{r} b_{1, i}^{(r)}(M)\right) D_{i} v_{1} \\
& +\frac{1}{s_{1}}\left(\sum_{r=1}^{m} s_{r} c_{1}^{(r)}(M)\right) v_{1}-D_{t} v_{1}=H_{1}(M) .
\end{aligned}
$$

ЗАмечАние 4 . Поскольку матрицы $\left[a_{1, i j}^{(r)}(M)\right] \in M_{n \times n} \forall r=\overline{1, m}$ системы (4) предполагаются положительно полуопределенными на множестве $G \cup \gamma(G)$, то при любом положительном фиксированном в условии (А.1) стохастическом векторе $p \in M_{m \times 1}$ из (5) выполнена оценка снизу:

$$
\frac{1}{s_{1}} \sum_{i, j=1}^{n}\left(\sum_{r=1}^{m} s_{r} a_{1, i j}^{(r)}(M)\right) \xi_{i} \xi_{j} \geqslant 0 \quad \forall M \in G \cup \gamma(G), \quad \forall|\xi|_{n}>0,
$$

и поэтому уравнение 2-го порядка (51) имеет неотрицательную характеристическую форму, т.е. является параболическим уравнением 2-го порядка, условие диссипативности для которого имеет вид

$$
\frac{1}{s_{1}} \sum_{r=1}^{m} s_{r} c_{1}^{(r)}(M) \leqslant 0 \quad \forall M \in G \cup \gamma(G) .
$$

ОпРЕДЕЛЕнИЕ 2 (ср. [1]-[5]). Для параболического уравнения 2-го порядка (51), заданного на ограниченном множестве $G \cup \gamma(G)$, справедлив слабый принuип минимума (СПМин) (соответственно слабый принцип максимума (СПМах)), если для любого суперпараболического (субпараболического) решения $v_{1}(M)$ класса (50) уравнения (51), т.е. при $H_{1}(M) \leqslant 0$ или $H_{1}(M)<0$ (соответственно $H_{1}(M) \geqslant 0$ или $\left.H_{1}(M)>0\right) \forall M=(x, t) \in G \cup \gamma(G)$ не сушествует точки $M_{0}=\left(x_{0}, t_{0}\right) \in G \cup \gamma(G)$ и полуокрестности

$$
U^{-}\left(M_{0}\right) \equiv \amalg_{\rho}\left(M_{0}\right) \cap\left\{t \leqslant t_{0}\right\} \equiv\left\{(x, t)|| x-x_{0} \mid<\rho, t_{0}-\rho<t \leqslant t_{0}\right\}
$$

таких, что выполнена оценка снизу:

$$
v_{1}(M) \geqslant v_{1}\left(M_{0}\right) \quad \forall(x, t)=M \in U^{-}\left(M_{0}\right) \subset G \cup \gamma(G),
$$

где либо $v_{1}\left(M_{0}\right)<0$ при $H_{1}\left(M_{0}\right) \leqslant 0$, либо $v_{1}\left(M_{0}\right) \leqslant 0$ при $H_{1}\left(M_{0}\right)<0$ (соответственно выполнена оценка сверху: $v_{1}(M) \leqslant v_{1}\left(M_{0}\right) \forall M \in U^{-}\left(M_{0}\right)$, где либо $v_{1}\left(M_{0}\right)>0$ при $H_{1}\left(M_{0}\right) \geqslant 0$, либо $v_{1}\left(M_{0}\right) \geqslant 0$ при $\left.H_{1}\left(M_{0}\right)>0\right)$. 
ОПРЕДЕЛЕНИЕ 3. Для параболической системы 2-го порядка (1) ( (4)), заданной на ограниченном множестве $G \cup \gamma(G) \subset \mathbb{R}_{x, t}^{n+1}$ и имеющей (см. (3)) решение

$$
u(M)=\left[\begin{array}{c}
u_{1}(M) \\
\ldots \ldots \\
u_{m}(M)
\end{array}\right] \in C_{x, t}^{(2,1)}(G \cup \gamma(G))
$$

при правой части $\Phi(M)=\left[\begin{array}{c}\Phi_{1}(M) \\ \ldots \ldots \\ \Phi_{m}(M)\end{array}\right]$ справедлив слабый (алгебраический) принцип минимума САПМин (соответственно САПМах), если:

1) матрицы (2) системы (4) удовлетворяют условию (А.1) определения 1 при фиксированном положительном стохастическом векторе $p>0$ и фиксированных векторах $f_{k} \in M_{m \times 1}(k=\overline{1, m})$ из (5);

2) для параболического уравнения 2-го порядка (51), соответствующего системе (4), где

$$
v_{1}(M)=\langle p, u(M)\rangle, \quad H_{1}(M)=\langle p, \Phi(M)\rangle,
$$

справедлив СПМин (СПМах) в смысле определения 2.

ТЕОРема 1 (о САПМин, САПМах для системы (4)). Пусть на множестве $G \cup \gamma(G) \subset \mathbb{R}_{x, t}^{n+1} \quad(n \geqslant 1)$ задана параболическая система 2-го порядка (4) ((1)), матрицы которой удовлетворяют условию (A.1) определения 1 с общими фиксированными стохастическим вектором $p>0$ и векторами $f_{k} \in M_{m \times 1}(k=\overline{1, m})$ из (5). Пусть (см. (3)) и(M) есть решение класса (54) системи (4).

Тогда векторно-матричному уравнению 2-го порядка (4) соответствует одно скалярное уравнение 2 -го порядка (51) для $v_{1}(M)=\langle p, u(M)\rangle$ класса (50) с правой частью $H_{1}(M)=\langle p, \Phi(M)\rangle$, параболическое в силу оченок (52) (при стохастическом векторе $p>0)$ с условием диссипативности (53).

Пусть $\forall M=(x, t) \in G \cup \gamma(G)$ выполнено одно из двух условий:

1) $H_{1}(M)<0 \quad(>0) u(53)$;

2) $H_{1}(M) \leqslant 0 \quad(\geqslant 0)$, но

$$
\frac{1}{s_{1}} \sum_{k=1}^{m} s_{k} c_{1}^{(k)}(M)<0 \quad \forall M \in G \cup \gamma(G) .
$$

Тогда для решения $v_{1}(M) \equiv\langle p, u(M)\rangle$ параболического уравнения 2-го порядка (51) справедлив СПМин (СПМах) в смысле определения 2, и потому для параболической системь 2-го порядка (1) справедлив САПМин (САПМах) на множестве $G \cup \gamma(G)$ в смысле определения 3 .

Доказательство теоремы 1 следует из теоремы о слабом принципе экстремума для одного параболического уравнения 2-го порядка с неотрицательной характеристической формой, доказываемой (см., например, [1]-[5]) в качественной теории параболических уравнений 2-го порядка. 
СлЕДСТВИЕ 1. Пусть на ограниченном множестве $G \cup \gamma(G)$ задана параболическая система 2-го порядка (4) ((1)), матричь которой удовлетворяют условию (A.1) определения 1 с общими фиксированным положительным стохастическим вектором $p>0$ и фиксированными векторами $f_{k} \in M_{m \times 1}$ $(k=\overline{1, m})$ из (5) при выполнении оценки

$$
\frac{1}{s_{1}} \sum_{i, j=1}^{n}\left(\sum_{r=1}^{m} s_{r} a_{1, i j}^{(r)}(M)\right) \xi_{i} \xi_{j}>0 \quad \forall M=(x, t) \in G \cup \gamma(G), \quad \forall|\xi|_{n}>0 .
$$

Пусть

$$
u=\left[\begin{array}{c}
u_{1} \\
\cdots \\
u_{m}
\end{array}\right] \in C(\bar{G}) \wedge C_{x, t}^{(2,1)}(G \cup \gamma(G))
$$

- решение системь (1), так что

$$
v_{1} \equiv\langle p, u\rangle \in C(\bar{G}) \wedge C_{x, t}^{(2,1)}(G \cup \gamma(G))
$$

- решение параболического уравнения 2-го порядка (51) с положительной характеристической формой. Пусть выполнено условие диссипативности (55) для уравнения (51) и решение $v_{1}$ уравнения (51) суперпараболично (субпараболично) на $G \cup \gamma(G)$, m.е.

$$
H_{1}(M) \equiv\langle p, \Phi(M)\rangle \leqslant 0(\geqslant 0) \quad \forall M \in G \cup \gamma(G) .
$$

Тогда

$$
\left(\left.v_{1}\right|_{\Gamma(G)} \geqslant 0\right) \Rightarrow\left(v_{1}(M) \geqslant 0 \quad \forall M \in \bar{G}\right)
$$

соответственно

$$
\left(\left.v_{1}\right|_{\Gamma(G)} \leqslant 0\right) \Rightarrow\left(v_{1}(M) \leqslant 0 \quad \forall M \in \bar{G}\right) .
$$

ДОКАЗАТЕЛЬСТВО. Пусть для определенности

$$
\left.v_{1}\right|_{\Gamma(G)} \geqslant 0
$$

где $v_{1}(M)$ - суперпараболическое решение параболического уравнения 2-го порядка (51), удовлетворяющего условию диссипативности (55). Докажем, что

$$
v_{1}(M) \geqslant 0 \quad \forall M \in \bar{G} .
$$

Допустим от противного, что $\exists M_{1} \in \bar{G}$ такая, что $v_{1}\left(M_{1}\right)<0$. Тогда в силу теоремы Вейерштрасса для функции $v_{1} \in C(\bar{G})$ сушествует точка $M_{0}=\left(x_{0}, t_{0}\right) \in \bar{G}$ такая, что $v_{1}\left(M_{0}\right)=\min _{\bar{G}} v_{1}<0$. В силу (59) $M_{0} \notin \Gamma(G)$, т.е. $M_{0} \in G \cup \gamma(G)$ является точкой локального отрищательного минимума суперпараболической функции $v_{1}$ класса (50), что противоречит в силу теоремы 1 СПМин для параболического уравнения 2-го порядка (51). 
СлЕДСТВИЕ 2. Пусть для системы (1) выполнены условия следствия 1 и и класса (57) (см. (3)) является решением однородной І-й краевой задачи для системь (4)

$$
\begin{aligned}
\mathscr{L} u(M)=0 & \forall M=(x, t) \in G \cup \gamma(G), \\
u_{r}(x, 0)=0 & \forall(x, 0) \in \bar{\gamma}_{0}(G), \quad r=\overline{1, m}, \\
u_{r}(M)=0 & \forall M \in \Sigma(G), \quad r=\overline{1, m} .
\end{aligned}
$$

Тогда

$$
v_{1}(M) \equiv\langle p, u(M)\rangle \equiv 0 \quad \text { в } \quad \bar{G},
$$

так что система функиий $\left\{u_{1}(M), \ldots, u_{m}(M)\right\}$ (компонент вектор-функции и) линейно зависима на множестве $\bar{G}$.

СлЕДСТВИЕ 3 (об усиленном СПМин (СПМах) для параболического уравнения 2 -го порядка (51)). Пусть на ограниченном множестве $G \cup \gamma(G)$ задано уравнение 2-го порядка (51) с положительной характеристической формой при выполнении оченки (56), для которого выполнено условие диссипативности (53). Пусть функция $v_{1}(M)$ класса (58) суперпараболична (субпараболична) на множестве $G \cup \gamma(G)$, m.е.

$$
L_{1} v_{1}(M) \leqslant 0(\geqslant 0) \quad \forall M=(x, t) \in G \cup \gamma(G) .
$$

Тогда

$$
\min _{\bar{G}} v_{1}=\min _{\Gamma(G)} v_{1} \quad n p u \quad \min _{\Gamma(G)} v_{1} \leqslant 0
$$

соответственно

$$
\max _{\bar{G}} v_{1}=\max _{\Gamma(G)} v_{1} \quad n p u \quad \max _{\Gamma(G)} v_{1} \geqslant 0
$$

и, в частности,

$$
\left(\left.v_{1}\right|_{\Gamma(G)} \geqslant 0\right) \Rightarrow\left(v_{1}(M) \geqslant 0 \quad \forall M \in \bar{G}\right)
$$

соответственно

$$
\left(\left.v_{1}\right|_{\Gamma(G)} \leqslant 0\right) \Rightarrow\left(v_{1}(M) \leqslant 0 \quad \forall M \in \bar{G}\right)
$$

Доказательство следствия 3 проводится по схеме доказательства соответствующего утверждения для уравнения теплопроводности из [5]. 
СлЕДСТВИЕ 4 (о единственности I-й краевой задачи для параболического уравнения 2-го порядка (51)). Пусть на ограниченном множестве $G \cup \gamma(G)$ задано уравнение 2-го порядка (51) с положстельной при выполнении оченки (56) характеристической формой, для которого вьполнено условие диссипативности (53).

Тогда может существовать не более одного решения $v_{1}(M)$ класса (58) I-й краевой задачи

$$
L_{1} v_{1}(M)=F(M) \quad \forall M=(x, t) \in G \cup \gamma(G)
$$

с начальныц условием

$$
v_{1}(x, 0)=\varphi(x) \quad \forall(x, 0) \in \bar{\gamma}_{0}(G)
$$

(әде $\left.\varphi \in C\left(\bar{\gamma}_{0}(G)\right)\right)$ при выполнении краевого условия 1-го рода

$$
v_{1}(M)=\psi(M) \quad \forall M \in \Sigma(G)
$$

(әде $\psi \in C(\Sigma(G)))$ и условия согласования

$$
\psi(x, 0)=\varphi(x) \quad \forall(x, 0) \in \bar{\gamma}_{0}(G) \cap \Sigma(G) .
$$

СЛЕДСТВИЕ 5. Пусть на ограниченном множестве $G \cup \gamma(G)$ задана параболическая система 2-го порядка (4) с положительными собственными значениями

$$
0<\lambda_{k, 1}^{(r)}(M) \leqslant \cdots \leqslant \lambda_{k, n}^{(r)}(M)<+\infty \quad \forall M \in G \cup \gamma(G) \quad(r, k=\overline{1, m}), \quad(67)
$$

для матриц (2) которой выполнено условие (А.1) теоремы 1 и выполнено условие диссипативности (53) для соответствующего системе (4) параболического уравнения 2-го порядка (51) (с положительной в силу (67) характеристической формой). Если вектор-функиия и (см. (3)) класса (57) является решением однородной I-й краевой задачи (60)-(62), то имеет место тождество (63), так что система $\left\{u_{1}(M), \ldots, u_{m}(M)\right\}$ компонент решения $u \in M_{m \times 1}$ задачи (60)-(62) линейно зависима на множестве $\bar{G}$.

ЗАМЕчАНИЕ 5. При выполнении условия (А.1) на коэффициенты (матрищ) системы (4) налагается $m-1$ (при $m \geqslant 2$ ) условий $\beta_{k}(M) \equiv 0 \forall k=\overline{2, m}$, что, естественно, сужает класс рассматриваемых систем (1), для которых справедлив САПМин (САПМах) теоремы 1 и следствий 1-5. Отметим попутно, что при выполнении условия (А.1) можно доказать лишь линейную зависимость функций $\left\{u_{1}(M), \ldots, u_{m}(M)\right\}$, являюшихся компонентами вектор-решения $u(M) \in M_{m \times 1}$ однородной I-й краевой задачи (60)-(62) для системы (4). 
ЗАмЕчАниЕ 6 . Ниже приводятся теоремы единственности решения I-й краевой задачи (60)-(62) в случаях $m=2$ и $m=3$.

ТЕОРЕма 2 (о единственности решения І-й краевой задачи для системы (1) при $m=2)$. Пусть на ограниченном множестве $G \cup \gamma(G) \subset \mathbb{R}_{x, t}^{n+1}(n \geqslant 1)$ задана параболическая система 2-го порядка (4) ((1)) при $m=2$ и положительных собственных значениях $(67)(r, k=1,2)$, матрицы (2) которой удовлетворяют условию (А.1) определения 1 с общими фиксированнымм стохастическим полохительным вектором $p=\left[\begin{array}{l}s_{1} \\ s_{2}\end{array}\right]>0 \quad\left(\right.$ әде $s_{1}+s_{2}=1, s_{k}>0 \quad(k=1,2)$ и $\left.s_{1} \neq \frac{1}{2}\right)$ и фиксированными векторами $f_{1}, f_{2} \in M_{2 \times 1}$ из (5). Пусть выполнены условие диссипативности (53) (при $m=2$ ), дополнительные оценки (параболичности)

$$
\begin{gathered}
\left(1-\frac{s_{2}}{s_{1}}\right) \sum_{i, j=1}^{n}\left(a_{2, i j}^{(2)}(M)-\frac{s_{2}}{s_{1}} a_{1, i j}^{(2)}(M)\right) \xi_{i} \xi_{j}>0 \\
\forall M \in G \cup \gamma(G), \quad \forall|\xi|_{n}>0
\end{gathered}
$$

и оценки (диссипативности)

$$
\left(1-\frac{s_{2}}{s_{1}}\right)\left(c_{2}^{(2)}(M)-\frac{s_{2}}{s_{1}} c_{1}^{(2)}(M)\right) \leqslant 0 \quad \forall M \in G \cup \gamma(G) .
$$

Тогда если вектор-функиия и (см. (3)) класса (57) (при $m=2$ ) является решением однородной I-й краевой задачи (60)-(62) для системы (1) (при $m=2)$, то имеет место тождество

$$
u(x, t) \equiv 0 \quad \text { \& } \quad \bar{G} .
$$

ДокАЗАТЕЛЬСТво. Пусть сушествует решение $u \in M_{2 \times 1}$ класса (57) (при $m=2$ ) однородной І-й краевой задачи $(60)-(62)$ для системы $(1)$ (при $m=2$ ). Тогда в силу выполнения условия (А.1) определения 1 для матрищ (2) системы (4) видим, что система (1) сводится к однородному уравнению 2-го порядка (51) при $m=2$ в $H_{1}(M)=\sum_{k=1}^{2} s_{k} \Phi_{k}(M)$, параболическому при выполнении оценки (56) для функции $v_{1}(M)=\sum_{k=1}^{2} s_{k} u_{k}(M)$ класса (58), являющейся решением однородной І-й краевой задачи $(64)$ при $F(M) \equiv 0,(65)$ при $\varphi(x) \equiv 0$ и (66) при $\psi(M) \equiv 0$. Поскольку выполнено условие диссипативности (53) (при $m=2$ ), то в силу следствия 4 имеем тождество (63), так что

$$
u_{1}(M) \equiv-\frac{s_{2}}{s_{1}} u_{2}(M) \quad \text { в } \bar{G} .
$$


Но тогда из 2-го скалярного уравнения системы (1) (при $r=2$ и $\Phi_{2} \equiv 0, m=2$ ) имеем при замене (71) уравнение

$$
\begin{aligned}
& \sum_{i, j=1}^{n}\left(a_{2, i j}^{(2)}(M)-\frac{s_{2}}{s_{1}} a_{1, i j}^{(2)}(M)\right) D_{i} D_{j} u_{2}(M) \\
& \quad+\sum_{i=1}^{n}\left(b_{2, i}^{(2)}(M)-\frac{s_{2}}{s_{1}} b_{1, i}^{(2)}(M)\right) D_{i} u_{2}(M) \\
& \quad+\left(c_{2}^{(2)}(M)-\frac{s_{2}}{s_{1}} c_{1}^{(2)}(M)\right) u_{2}(M)-\left(1-\frac{s_{2}}{s_{1}}\right) D_{t} u_{2}(M)=0 .
\end{aligned}
$$

Заметим, что условие $s_{1} \neq 1 / 2$ для стохастического вектора $p \in M_{2 \times 1}$ обеспечивает присутствие в уравнении (72) члена с временной производной $D_{t} u_{2}(M)$. Но тогда уравнение (72) будет параболическим при вьполнении оценки (68), причем условие диссипативности для уравнения (72) имеет вид (69). Но тогда функция $u_{2}(M)$ класса $(57)$ является решением однородного параболического уравнения 2-го порядка (72) с положительной характеристической формой и при выполнении условия диссипативности (69) обеспечивает применение к функции $u_{2}$ усиленного слабого принципа экстремума (ср. следствия 3 и 4 ) на множестве $G \cup \gamma(G)$. А поскольку выполнено начальное условие $u_{2}(x, 0)=0 \forall(x, 0) \in \bar{\gamma}_{0}(G)$ (см. (61) при $r=m=2$ ) и однородное краевое условие (62) (при $r=m=2$ ), то в силу обычной теоремы единственности (см. следствие 3 ) решения І-й краевой задачи для одного параболического уравнения 2-го порядка (72) имеем тождество

$$
u_{2}(M) \equiv 0 \quad \text { в } \bar{G},
$$

откуда в силу (71) имеем

$$
u_{1}(M) \equiv-\frac{s_{2}}{s_{1}} u_{2}(M) \equiv 0 \quad \text { в } \bar{G}
$$

(при $s_{1} \neq 1 / 2$ ), что и дает тождество (70) теоремы 2.

Пусть теперь матрищы (47)-(49) удовлетворяют условию (А.2) (при $m=3$ ) определения 1 . Тогда в силу (38) леммы 2 и (45) замечания 3 , получаем, в частности, скалярное уравнение 2-го порядка для функции

$$
v_{2}=\frac{1}{s_{2}}\left(\sum_{r=1}^{3} s_{r} u_{r}-u_{1}\right) \in C_{x, t}^{(2,1)}(G \cup \gamma(G))
$$


при

$$
\begin{gathered}
H_{2}(M)=\frac{1}{s_{2}}\left(\sum_{r=1}^{3} s_{r} \Phi_{r}(M)-\Phi_{1}(M)\right) \\
L_{2} v_{2} \equiv \frac{1}{s_{2}} \sum_{i, j=1}^{n}\left(a_{2, i j}^{(1)}(M)-\sum_{r=1}^{3} s_{r} a_{2, i j}^{(r)}(M)\right) D_{i} D_{j} v_{2} \\
+\frac{1}{s_{2}} \sum_{i=1}^{n}\left(b_{2, i}^{(1)}(M)-\sum_{r=1}^{3} s_{r} b_{2, i}^{(r)}(M)\right) D_{i} v_{2} \\
+\frac{1}{s_{2}}\left(c_{2}^{(1)}(M)-\sum_{r=1}^{3} s_{r} c_{2}^{(r)}(M)\right) v_{2}-D_{t} v_{2}=H_{2}(M) \\
\forall M \in G \cup \gamma(G)
\end{gathered}
$$

ЗАмЕчАниЕ 6. При выполнении условия (А.2) определения 1 для матрищ (2) $(m=3)$ системы $(4)$ уравнение (73) имеет положительную характеристическую форму при выполнении оценки снизу

$$
\begin{gathered}
\frac{1}{s_{2}} \sum_{i, j=1}^{n}\left(a_{2, i j}^{(1)}(M)-\sum_{r=1}^{3} s_{r} a_{2, i j}^{(r)}(M)\right) \xi_{i} \xi_{j}>0 \\
\forall M \in G \cup \gamma(G), \quad \forall|\xi|_{n}>0
\end{gathered}
$$

с условием диссипативности

$$
\frac{1}{s_{2}}\left(c_{2}^{(1)}(M)-\sum_{r=1}^{3} s_{r} c_{2}^{(r)}(M)\right) \leqslant 0 \quad \forall M \in G \cup \gamma(G) .
$$

Тогда для параболического уравнения 2-го порядка (73) выполнен усиленный слабый принщип экстремума (ср. следствие 3 и [5]) и имеет место теорема 3.

ТЕОРемА 3 (о единственности решения I-й краевой задачи для уравнения (73)). Пусть на ограниченном множсестве $G \cup \gamma(G) \subset \mathbb{R}_{x, t}^{n+1}(n \geqslant 1)$ задано уравнение 2-го порядка (73) с положительной характеристической формой при выполнении оценки (74) и выполнено условие диссипативности (75).

Тогда может существовать не более одного решения $v_{2}(M)$ класса (55) I-й краевой задачи

$$
\begin{aligned}
L_{2} v_{2}(M) & =F(M) & & \forall M \in G \cup \gamma(G), \\
v_{2}(x, 0) & =\varphi(x) & & \forall(x, 0) \in \bar{\gamma}_{0}(G), \\
v_{2}(M) & =\psi(M) & & \forall M \in \Sigma(G),
\end{aligned}
$$

$u$, в частности, если функиия $v_{2}(M)$ класса (58) является решением однородной I-й краевой задачи (76) при $F \equiv 0,(77)$ при $\varphi \equiv 0 u$ (78) при $\psi \equiv 0$, то имеет место тождество

$$
v_{2}(M) \equiv 0 \quad \text { в } \quad \bar{G}
$$


TЕОРЕма 4 (о единственности решения І-й краевой задачи для системы (1) ( (4)) при $m=3)$. Пусть на ограниченном множестве $G \cup \gamma(G) \subset \mathbb{R}_{x, t}^{n+1}(n \geqslant 1)$ задана параболическая система 2-го порядка (1) ((4)) (әде $m=3$ ) при положительных собственных значениях $(67)(r, k=\overline{1,3}, \quad m=3)$, матрииы (2) которой удовлетворяют одновременно условиям (А.1) и (А.2) определения 1 с общими фиксированным положительным стохастическим векпором $p=\left[\begin{array}{l}s_{1} \\ s_{2} \\ s_{3}\end{array}\right]>0 \quad\left(\right.$ əде $\left.\sum_{k=1}^{3} s_{k}=1, \quad s_{k}>0, \quad k=\overline{1,3}, u s_{3} \neq s_{2}\right) u$ фиксированными векторами $f_{k} \in M_{3 \times 1} \quad(k=\overline{1,3})$ из (5). Пусть выполнены оценки (56), (74), условия диссипативности (53), (75) и дополнительное условие (параболичности) при $s_{2} \neq s_{3}$

$$
\begin{gathered}
\left(1-\frac{s_{2}}{s_{3}}\right) \sum_{i, j=1}^{n}\left(a_{2, i j}^{(2)}(M)-\frac{s_{2}}{s_{3}} a_{3, i j}^{(2)}(M)\right) \xi_{i} \xi_{j}>0 \\
\forall M=(x, t) \in G \cup \gamma(G), \quad \forall|\xi|_{n}>0,
\end{gathered}
$$

а также дополнительное условие (диссипативности) при $s_{2} \neq s_{3}$

$$
\left(1-\frac{s_{2}}{s_{3}}\right)\left(c_{2}^{(2)}(M)-\frac{s_{2}}{s_{3}} c_{3}^{(2)}(M)\right) \leqslant 0 \quad \forall M \in G \cup \gamma(G) .
$$

Тогда если вектор-функция $и \in M_{3 \times 1}$ из (3) класса (57) является решением однородной I-й краевой задачи (60)-(62) для параболической системы 2-го порядка (1) (при $m=3)$, то

$$
u(x, t) \equiv 0 \quad \text { \& } \quad \bar{G} .
$$

ДокАЗАТЕЛЬСтво. Поскольку для матрищ (2) системы (4) выполнены условия (А.1) и (А.2) определения 1 , то системе (1) (с $\Phi_{r} \equiv 0, r=\overline{1,3}$ ) соответствуют два однородных скалярных уравнения 2-го порядка: (51) (для $v_{1}$ при $m=3$ и $\left.H_{1}(M) \equiv 0\right)$ и $(73)$ (для $v_{2}$ при $m=3$ и $H_{2}(M) \equiv 0$ ), для которых выполнены условия диссипативности (53) и (75), причем уравнения (51) и (73) параболичны в силу $(67),(56),(74)$. Тогда в силу (63), следствия 5 и тождества (79) теоремы 3 имеем тождества

$$
\begin{gathered}
v_{1}(M)=\sum_{r=1}^{3} s_{r} u_{r}(M) \equiv 0, \\
v_{2}(M)=\frac{1}{s_{2}}\left(v_{1}(M)-u_{1}(M)\right) \equiv 0 \quad \text { в } \bar{G}
\end{gathered}
$$

так что $u_{1}(M) \equiv 0$ в $\bar{G}$. Но тогда имеем

$$
u_{1}(M) \equiv 0 \equiv s_{2} u_{2}(M)+s_{3} u_{3}(M),
$$


т.е.

$$
u_{1}(M) \equiv 0, \quad u_{3}(M) \equiv-\frac{s_{2}}{s_{3}} u_{2}(M) \quad \text { в } \bar{G} .
$$

Подставляя во второе $(r=2)$ однородное скалярное уравнение системы (1) (при $\left.\Phi_{2} \equiv 0, m=3\right) u_{1} \equiv 0, u_{3} \equiv-\frac{s_{2}}{s_{3}} u_{2}$, получаем для компоненты $u_{2}$ класса $(57)$ уравнение 2-го порядка

$$
\begin{aligned}
& \sum_{i, j=1}^{n}\left(a_{2, i j}^{(2)}(M)-\frac{s_{2}}{s_{3}} a_{3, i j}^{(2)}(M)\right) D_{i} D_{j} u_{2} \\
& +\sum_{i=1}^{n}\left(b_{2, i}^{(2)}(M)-\frac{s_{2}}{s_{3}} b_{3, i}^{(2)}(M)\right) D_{i} u_{2} \\
& +\left(c_{2}^{(2)}(M)-\frac{s_{2}}{s_{3}} c_{3}^{(2)}(M)\right) u_{2}-\left(1-\frac{s_{2}}{s_{3}}\right) D_{t} u_{2}=0 \\
& \forall M=(x, t) \in G \cup \gamma(G) \quad\left(s_{2} \neq s_{3}\right),
\end{aligned}
$$

параболическое при выполнении дополнительной оценки (80). При выполнении условия диссипативности (81) имеем, учитывая однородные условия (61), (62) (при $r=2, m=3)$,

$$
\begin{array}{ll}
u_{2}(x, 0)=0 & \forall(x, 0) \in \bar{\gamma}_{0}(G), \\
u_{2}(M)=0 & \forall M \in \Sigma(G) .
\end{array}
$$

Но тогда к однородной І-й краевой задаче (83), (84) применима обычная теорема единственности (основанная на усиленном принципе экстремума из [5] и следствии 3$)$, дающая тождество $u_{2}(M) \equiv 0 \equiv u_{3}(M) \equiv u_{1}(M)$ в $\bar{G}$. Этим тождество (82) теоремы 4 доказано.

ЗАмЕЧАНИЕ 7. Метод спуска, использованный при доказательстве теоремы 4 в случае $m=3$, позволяет доказать аналогичную теорему единственности и в случае $m \geqslant 4$ при введении условий $($ А. $k$ ) для $k=\overline{1, m-1}$, т.е. равенств

$$
\beta_{(k-1) m+r}=0 \quad \forall r=\overline{1, k-1}, \overline{k+1, m} \quad(\forall r \neq k),
$$

с последующим выражением компонент $\left\{u_{1}, \ldots, u_{m}\right\}$ решения системы (1) через одну из них $u_{i}$ и сведением $i$-го уравнения системы (1) (при $r=i$ ) к одному параболическому уравнению 2-го порядка для $u_{i}$.

ЗАмЕчАнИЕ 8. Вьполнение условия (А.1) определения 1 в теореме 1 для системы (1) ((4)) сводится к выполнению при $k=\overline{2, m} \forall M \in G \cup \gamma(G)$ тождеств

$$
\begin{aligned}
\frac{1}{s_{k}} \sum_{r=1}^{m} s_{r} a_{k, i j}^{(r)}(M) & \equiv \frac{1}{s_{k-1}} \sum_{r=1}^{m} s_{r} a_{k-1, i j}^{(r)}(M) \stackrel{\text { def }}{=} \mu_{i j}(M), \\
\frac{1}{s_{k}} \sum_{r=1}^{m} s_{r} b_{k, i}^{(r)}(M) & \equiv \frac{1}{s_{k-1}} \sum_{r=1}^{m} s_{r} b_{k-1, i}^{(r)}(M) \stackrel{\text { def }}{=} \mu_{i}(M),
\end{aligned}
$$


где $i, j=\overline{1, n}$,

$$
\frac{1}{s_{k}} \sum_{r=1}^{m} s_{r} c_{k}^{(r)}(M) \equiv \frac{1}{s_{k-1}} \sum_{r=1}^{m} s_{r} c_{k-1}^{(r)}(M) \stackrel{\text { def }}{=} \mu(M)
$$

допускаюших векторно-матричную запись (см. (2))

$$
\begin{gathered}
p^{T} A_{i j}(M)=\mu_{i j}(M) p^{T}, \quad p^{T} B_{i}(M)=\mu_{i}(M) p^{T}, \quad i, j=\overline{1, n} \\
p^{T} C(M)=\mu(M) p^{T}
\end{gathered}
$$

где $p=\left[\begin{array}{c}s_{1} \\ \ldots \\ s_{m}\end{array}\right] \in M_{m \times 1}$ взят из $(5)$ и $p^{T}=\left[s_{1} \ldots s_{m}\right] \in M_{1 \times m}$. Но выполнение (85)

означает, что матрицы $A_{i j}(M), B_{i}(M), C(M) \in M_{m \times m}(i, j=\overline{1, n})$ системы (4) имеют общий левый собственный вектор $p \in M_{m \times 1}$ из (5) с соответствуюшими собственными значениями $\mu_{i j}(M), \mu_{i}(M), \mu(M)(i, j=\overline{1, n})$. Умножая систему (4) слева на $p^{T}$, получаем в силу (85) уравнение (51) для функции $v_{1}(M)=\langle p, u(M)\rangle$ из (50), допускающую запись в виде

$$
\begin{gathered}
\sum_{i, j=1}^{n} \mu_{i j}(M) D_{i} D_{j} v_{1}(M)+\sum_{i=1}^{n} \mu_{i}(M) D_{i} v_{1}(M) \\
+\mu(M) v_{1}(M)-D_{t} v_{1}(M)=\langle p, \Phi(M)\rangle .
\end{gathered}
$$

ПРИмеР. Рассмотрим систему (1) при $m=2, n \geqslant 2$ вида

$$
\begin{aligned}
\mathscr{L}_{1}(u) & \equiv \Delta u_{1}+\Delta u_{2}+\sum_{k=1}^{2} c_{k}^{(1)}(M) u_{k}-D_{t} u_{1}=\Phi_{1}(M), \\
\mathscr{L}_{2}(u) & \equiv \sum_{k=1}^{2}\left(\sum_{i=1}^{n} a_{k, i i}^{(2)}(M) D_{i} D_{i} u_{k}+c_{k}^{(2)}(M) u_{k}\right)-D_{t} u_{2} \\
& =\Phi_{2}(M) \quad \forall M \in G \cup \gamma(G)
\end{aligned}
$$

и подберем $a_{k, i i}^{(2)}(M)>0 \quad(k=1,2 ; i=\overline{1, n})$ такими, чтобы для системы $(87)$ выполнялись условия (А.1) теоремы 1 . Перепишем систему (87) в виде (см. (2)-(4))

$$
\mathscr{L}(u) \equiv \sum_{i=1}^{n} A_{i i}(M) D_{i} D_{i} u+C(M) u-D_{t} u=\Phi(M),
$$

где положено

$$
\begin{aligned}
A_{i i}(M) & =\left[\begin{array}{cc}
1 & 1 \\
a_{1, i i}^{(2)}(M) & a_{2, i i}^{(2)}(M)
\end{array}\right], \\
C(M) & =\left[\begin{array}{ll}
c_{1}^{(1)}(M) & c_{2}^{(1)}(M) \\
c_{1}^{(2)}(M) & c_{2}^{(2)}(M)
\end{array}\right] \in M_{2 \times 2}, \quad i=\overline{1, n} .
\end{aligned}
$$


Если матрицы $A_{i i}(M), C(M) \in M_{2 \times 2}$ удовлетворяют условию (А.1) теоремы 1 с общим вектором $p \in M_{2 \times 1}$ из (5), то в силу замечания 8 вектор $p$ является обшим левым собственным вектором для этих матрищ, так что имеет место (85) для $A_{i i}(M)$ и $C(M)$. Но тогда для собственных значений этих матриц из (85), (89) имеем

$$
\begin{gathered}
\mu_{i i}(M) \stackrel{\text { def }}{=} 1+\frac{s_{2}}{s_{1}} a_{1, i i}^{(2)}(M) \equiv \frac{1}{s_{2}}\left(s_{1}+s_{2} a_{2, i i}^{(2)}(M)\right)>0, \quad i=\overline{1, n}, \\
\mu(M) \stackrel{\text { def }}{=} c_{1}^{(1)}(M)+\frac{s_{2}}{s_{1}} c_{1}^{(2)}(M) \equiv \frac{1}{s_{2}}\left(s_{1} c_{2}^{(1)}(M)+s_{2} c_{2}^{(2)}(M)\right),
\end{gathered}
$$

и поэтому в системе (88) должно быть (после выражения из (90) $a_{2, i i}^{(2)}$ через $a_{1, i i}^{(2)}$ и $c_{2}^{(2)}$ через $\left.c_{1}^{(1)}, c_{2}^{(1)}, c_{1}^{(2)}\right)$

$$
A_{i i}(M)=\left[\begin{array}{cc}
1 & 1 \\
a_{1, i i}^{(2)}(M) & 1-\frac{s_{1}}{s_{2}}+\frac{s_{2}}{s_{1}} a_{1, i i}^{(2)}(M)
\end{array}\right] \in M_{2 \times 2}, \quad i=\overline{1, n}
$$

(где при выполнении оценок снизу

$$
a_{1, i i}^{(2)}(M)>0, \quad 1+\frac{s_{2}}{s_{1}} a_{1, i i}^{(2)}(M)>\frac{s_{1}}{s_{2}}>0
$$

нижняя строка $A_{i i}(M)$ состоит из положительных элементов), и

$$
A_{i i}(M)=\left[\begin{array}{cc}
c_{1}^{(1)}(M) & c_{2}^{(1)}(M) \\
c_{1}^{(2)}(M) & c_{1}^{(1)}(M)+\frac{s_{2}}{s_{1}}\left(c_{1}^{(2)}(M)-c_{2}^{(1)}(M)\right)
\end{array}\right] \in M_{2 \times 2} .
$$

Так найденной системе (88) соответствует скалярное параболическое (см. (90)) уравнение 2-го порядка (51) для $v_{1}=\langle p, u\rangle \in C_{x, t}^{2,1}(G \cup \gamma(G))$ вида (86) (см. (90))

$$
\sum_{i=1}^{n} \mu_{i i}(M) D_{i} D_{i} v_{1}+\mu(M) v_{1}=\langle p, \Phi(M)\rangle
$$

с условием диссипативности

$$
\mu(M) \equiv c_{1}^{(1)}(M)+\frac{s_{2}}{s_{1}} c_{1}^{(2)}(M) \leqslant 0 \quad \forall M \in G \cup \gamma(G) .
$$

В силу теоремы 1 найденная система (87) ((88)) обладает СПМин (СПМах) в смысле определения 2 . 


\section{Список литературы}

1. Ильин A. М., Калашников A. С., Олейник О. А. Линейные уравнения второго порядка параболического типа // УМН. 1962. Т. 17. № 3. С. 3-146.

2. Ладыженская О. А., Солонников В. А., Уральцева Н. Н. Линейные и квазилинейные уравнения параболического типа. М.: Наука, 1967.

3. Фридман A. Уравнения с частными производными параболического типа. М.: Мир, 1968.

4. Камынин Л.И., Химченко Б. Н. Теоремы Жиро о временной производной для параболического оператора второго порядка // Вестн. МГУ. Сер. матем., мех. 1977. №5. C. $45-53$.

5. Тихонов A.Н., Самарский А. А. Уравнения математической физики. М.: Наука, 1972.

6. Мазъя В. Г., Кресин Г. И. О принципе максимума для сильно эллиптических и параболических систем второго порядка с постоянными коэффициентами // Матем. сб. 1964. T. 125. № 4. C. 458-480.

7. Protter M. H., Weinberger H. F. Maximum principle in differential equations. N.J., 1967.

8. Alikakos $N$. Quantative maximum principle and strongly coupled gradientlike reaction-diffusion systems // Proceedings of the Royal Sociaty of Edinburg. 1983. V. 94. № 3-4. P. 265-284.

9. Сабитов К. Б. Экстремальные свойства решений одного класса параболических систем и их применения // Дифференц. уравн. 1990. Т. 26. № 2. С. 287-297.

10. Хорн Р., Джсонсон Ч. Матричный анализ. М.: Мир, 1989.

Поступило в редакцию

20.XI. 1995 